\title{
Living with mental illness: quality or continuity of life Aleksandar Janca
}

\author{
Address: School of Psychiatry and Clinical Neurosciences, University of Western Australia, Perth, Australia \\ from International Society on Brain and Behaviour: 3rd International Congress on Brain and Behaviour \\ Thessaloniki, Greece. 28 November - 2 December 2007 \\ Published: 17 April 2008 \\ Annals of General Psychiatry 2008, 7(Suppl I):S45 doi:I0.II86/I744-859X-7-SI-S45
}

This abstract is available from: http://www.annals-general-psychiatry.com/content/7/SI/S45

(c) 2008 Janca; licensee BioMed Central Ltd.

According to the Australian Study of Low Prevalence Disorders [1], a great majority of people with chronic and severe mental illness such as schizophrenia have harsh and unfulfilling lives: $75 \%$ are single, $30 \%$ live alone, $35 \%$ have no contact with relatives, $75 \%$ have no friend and $85 \%$ are reliant on welfare benefits. In the year prior to the study, $16 \%$ of them had attempted suicide, $18 \%$ had been a victim of violence, $15 \%$ felt unsafe where they lived, $13 \%$ needed police or legal assistance and $10 \%$ had been arrested. Nevertheless, when asked about their quality of life $60 \%$ of these people reported that they were mostly satisfied with their current level of independence and $44 \%$ were mostly satisfied with their life as a whole in the past year.

This somewhat paradoxical finding points toward a limited applicability of the concept and measurement of quality of life in people living with chronic and severe mental illness. In an attempt to resolve this paradox, we have developed a novel concept of "continuity of life" and defined it as an individual's ability continue with activities, plans and hopes in spite of an event or process such as chronic and severe mental illness. The concept has been accompanied by a new assessment instrument entitled the Continuity of Life Interview (COLI), which covers twelve domains including some that are typically missed by the quality of life measurements (e.g. access to material possessions and fulfilment of civic duties and responsibilities). The COLI assessment process focuses on the present state and future plans of the respondents, rather then on their past $[2,3]$.

The COLI was extensively evaluated in several studies spanning different types of patients, settings and cultures. Patients understood the concept and its domains well and often expressed the view that the COLI interview gave them the opportunity to discuss aspects of their mental ill- ness that are often disregarded during the treatment process. The COLI proved to be a cross-culturally appropriate and reliable tool with kappa coefficients of 0.845 and 0.451 for inter-rater and test-retest reliability, respectively. Furthermore, it has proven to be a suitable alternative and/or complement to most currently used quality of life measures. The COLI is now available to all potentially interested users and we hope that this presentation will prompt further translation, evaluation and application of this novel instrument.

\section{References}

I. Jablensky A, McGrath J, Herrman H, Castle D, Gureje O, Evans M, Carr V, Morgan V, Korten A, Harvey C: Psychotic disorders in urban areas: an overview of the Study on Low Prevalence Disorders. Aust N Z J Psychiatry 2000, 34:22I-236.

2. Janca A, Cooper JE: 'Measurement of some novel concepts in psychiatry. World Psychiatry 2002, 2:107-108.

3. Janca $A$, Ventouras J: (2005) Measurement in psychiatry: novel concepts and instruments. In Advances in Psychiatry Vol. II Edited by: Christodoulou GN. World Psychiatric Association, Athens; 2005:89-93. 